\title{
Efficacy of Brinzolamide in the Initial Management of Acute Primary Angle Closure in Chinese: a Randomized Controlled Trial
}

guang chen ( $\nabla$ cheng2021cn@163.com )

affiliated hospital of hebei university

\section{Xinming Peng}

affilited hospital of hebei university

Jun Li

affiliated hospital of hebei university

\section{Peng Chen}

affiliated hospital of hebei university

Jing Wang

affiliated hospital of hebei university

\section{Research Article}

Keywords: acute primary angle closure, brinzolamide, sequential treatment, pressure-lowering, casecontrol study

Posted Date: March 9th, 2021

DOl: https://doi.org/10.21203/rs.3.rs-230155/v1

License: (c) (i) This work is licensed under a Creative Commons Attribution 4.0 International License. Read Full License

Version of Record: A version of this preprint was published at Journal of Clinical Pharmacy and Therapeutics on January 13th, 2022. See the published version at https://doi.org/10.1111/jcpt.13609. 


\section{Abstract}

Objective: Since comprehensive medication has an important role in the initial management of patients presenting with acute primary angle closure, it is necessary to analyze the effect of each drug on alleviating the disease. This study aimed to evaluate the intraocular pressure-lowering effect of brinzolamide in the sequential treatment of acute primary angle closure.

Methods: In this randomized double-blind controlled trial, a total of 131 eyes of 125 consecutive patients who presented with their first episode of acute primary angle closure were recruited and received sequential treatment. The patients were randomized to receive either brinzolamide or normal saline as a placebo. The primary outcomes were decreased intraocular pressure, success rate, and treatment time.

Results: There was no statistically significant difference in the decreased level of intraocular pressure between the two groups at 6,12 , or 24 hours after the start of treatment (P-values were $0.526,0.206$, and 0.130 , respectively). The success rate and treatment time were also not significantly different between the groups. No adverse side effects of brinzolamide were observed in the brinzolamide group.

Conclusions: In patients with a first episode of acute primary angle closure, brinzolamide did not improve the effectiveness of the sequential treatment for reducing the intraocular pressure levels or shortening the treatment time within the first 24 hours of initiating therapy.

\section{Introduction}

Acute primary angle-closure glaucoma (PACG) accounts for nearly half of the blindness cases attributed to glaucoma worldwide.[1] The American Academy of Ophthalmology refers to acute primary angle closure (APAC) as an acute attack of PACG.[2] It is caused by a sudden occlusion of the angle by peripheral iris tissue, resulting in an abrupt rise in the intraocular pressure (IOP). Patients with APAC often have ocular pain, blurred vision, and headache. Persistently high IOP levels can result in different degrees of impairment of intraocular tissue and, ultimately, cause irreversible damage to visual function.[3]

Rapid reduction of the IOP is an important initial step in APAC treatment. The use of topical and systemic IOP-lowering medication is usually sufficient to achieve initial IOP control. Since comprehensive medication has an important role in the initial management of patients presenting with APAC, it is necessary to analyze the effect of each drug on alleviating the disease. One of the indications of brinzolamide is ocular hypertension. Thus, this study was undertaken to evaluate brinzolamide's IOPlowering effect on the sequential treatment of patients with APAC in whom conservative medication alone was insufficient to lower the IOP. Immediate anterior chamber paracentesis (ACP) and argon laser peripheral iridoplasty (ALPI) were performed with the patients' consent.[4]

\section{Subjects And Methods}


The study was a randomized controlled trial approved by the Institutional Review Board of our hospital. This study was conducted in accordance with the Declaration of Helsinki and approved by the ethics committee of our hospital. All patients consent to participate in this study.

The study inclusion criteria were: (1) age greater than 40 years and ability to provide informed consent; (2) diagnosed with APAC and experiencing APAC for the first time (APAC was defined based on the following criteria: ocular or periocular pain; nausea and/or vomiting; the presence of at least three of the following signs: corneal epithelial oedema, mid-dilated unreactive pupil, a shallow anterior chamber, or IOP levels of $40 \mathrm{mmHg}$ or greater as measured by the rebound tonometer; (3) no treatment received before the study; and (4) consent to ACP or ALPI treatment if drug therapy failed. The exclusion criteria were: (1) pupillary block was not the main mechanism of angle closure (as determined clinically); (2) patients with only a single eye; (3) history of ophthalmic disorders that may have a persistent effect on the structure or function of the drainage angle; and (4) other systemic diseases contraindicated for brinzolamide.

A total of 125 consecutive patients who presented with a first episode of APAC were recruited. After signing their informed consent, the patients were randomized into one of two treatment groups using a random number table. Each patient in the brinzolamide group was administered drops of topical brinzolamide (1\%) three times a day after beginning treatment. In the placebo group, drops of normal saline were administered three times a day as the placebo. Neither the front-line ophthalmologists who diagnosed the APAC nor the patients were aware of the treatment assignment. For patients with bilateral attack, both eyes were randomized together for the same treatment, i.e., either bilateral brinzolamide or placebo only. Documentation of visual acuity (VA), IOP, corneal edema, and pupillary size was performed before treatment.

In order to control the variables and avoid delaying the treatment, all of the APAC eyes (including the brinzolamide group and the placebo group) were treated with sequential treatment: (1) In the first hour, topical pilocarpine (4\%) was administered at one drop every 10 minutes, topical prednisolone acetate (1\%) was given at one drop every 30 minutes, and $250 \mathrm{~mL}$ of $20 \%$ intravenous mannitol was administered. After the first hour, pilocarpine was administered at one drop every two hours, and topical prednisolone acetate was given at one drop every four hours until the IOP returned to normal. (2) If the IOP remained greater than or equal to $30 \mathrm{mmHg}$ after six hours, ACP was performed under topical anesthesia. Whether to give ALPI depended on the effect of the ACP because of the severe corneal edema and shallow anterior chamber. It might be carried out after ACP because the corneal puncture orifice could be pressed to release part of the aqueous humor and the corneal edema was reduced temporarily.

The follow-up time was 24 hours after beginning the treatment. For both groups of patients, the IOP, VA, corneal edema, pupillary size, time of clinical symptom remission, total amount of mannitol, and number of patients who underwent ACP or ALPI were recorded. The IOP was measured by a masked investigator (who was unaware of the patient group status) at 6 hours, 12 hours, and 24 hours after treatment began. The criteria for successful treatment were determined as follows: (1) IOP less than $30 \mathrm{~mm} \mathrm{Hg}$; (2) 
reduction of corneal edema so that, at minimum, the iris details were not obscured; (3) pupil diameter less than $4 \mathrm{~mm}$. The eyes in remission were maintained on topical pilocarpine four times daily until the definitive treatment of laser peripheral iridotomy could be performed.

The above findings, data, and treatment side effects were recorded on a data sheet designed for this study. A masked statistician analyzed the data.

\section{Statistical Analysis}

The statistical analysis was performed using IBM SPSS Statistics 24.0. The categorical data were expressed in percentages and compared by a Pearson chi-squared test. The numerical data were expressed as the mean \pm sd. The IOP before treatment (normal distribution) was compared by an independent samples t-test. The reduction of the IOP after treatment and the time needed to relieve the crisis (non-normal distribution) were compared by a Mann-Whitney $U$ test. Statistical significance was accepted at $P \otimes 0.05$.

\section{Results}

The study was conducted on 131 eyes of 125 patients who were randomized into two groups: the brinzolamide group (63 eyes) and the placebo group (68 eyes). No patient dropped out of the study. The patients' ages ranged from 42 to 84 years (mean age: $64 \pm 9$ years). Table 1 displays the patients' demographics. There was no statistically significant difference between the two treatment groups regarding patient age, sex, laterality of the eye, or IOP before treatment (all P $₫ 0.05$ ).

Table 2 and Figure 1 show the mean IOP for all time intervals in both groups. Compared with their IOP baseline, both groups showed a statistically significant decrease in the IOP at all follow-up intervals (all $P$ $\otimes 0.05)$.

The reductions in the IOP of the two groups after treatment are presented in Table 3 and presented graphically in Figure 2. Using the Mann-Whitney $U$ test, there were no statistically significant differences in the reduction of IOP at all follow-up intervals between the two treatment groups (all P $\otimes 0.05$ ).

The success rates of sequential treatment at each time interval are presented in Table 4, and a KaplanMeier survival analysis showing the cumulative probabilities of the success rates for the two groups is presented in Figure 3. There were no statistically significant differences in the efficacy rates at any followup intervals between the two treatment groups (all $P \otimes 0.05$ ).

Among the 123 eyes (60 in the brinzolamide group and 63 in the placebo group) treated successfully, the clinical symptom remission time was $5.98 \pm 3.74$ hours in the brinzolamide group and $6.26 \pm 4.13$ hours in the placebo group, which was not statistically significantly different $(U=0.283, P=0.778)$.

There were no statistically significant differences in the total amount of mannitol, the number of patients who underwent ACP, or the number of patients who underwent ALPI, as presented in Table 5. 
Among the eight eyes with uncontrolled APAC after 24 hours of sequential treatment, three eyes were ultimately treated successfully by conservative treatment, and five eyes eventually underwent emergency trabeculectomy. No serious eye complications occurred during the sequential treatment in either group. Conjunctival hemorrhage occurred in 13 patients (27.1\%) after ACP, and one case developed localized corneal endothelial damage after ALPI. Taste abnormalities occurred in nine people (14.8\%). No adverse drug reaction related to brinzolamide occurred in the brinzolamide group.

\section{Discussion}

Acute primary angle closure is a potentially blinding ocular condition, occurring with high incidence in East Asians.[5,6] It has been reported that the number of PACG patients is expected to increase to 34 million people by 2040.[7] The primary goals of APAC treatment include relieving pupil block, opening the anterior chamber angle, and reducing the IOP. Laser peripheral iridotomy is the first-line therapy in eyes with primary angle-closure disease,[8] but eyes with APAC are unsuitable for immediate laser peripheral iridotomy because of corneal edema, iris hyperemia, and a shallow anterior chamber.[9,10] Topical and systemic IOP-lowering medications, which are used as a first-line drug usually, involve a topical ß-blocker, topical miotic agent, carbonic anhydrase inhibitor, oral hyperosmotic agent, and intravenous hyperosmotic agent.

Brinzolamide eye drops are a classic drug for treating open-angle glaucoma and are also a commonly used drug in the treatment of PACG. However, this study found no statistically significant difference between the brinzolamide group and placebo group in any stage of the sequential APAC treatment. There are several possible reasons for this finding: (1) In terms of pathogenesis, in the case of acute angle closure, brinzolamide can only reduce the generation of aqueous humor and has no effect on the aqueous humor outflow. In other words, brinzolamide only creates conditions for the action of pilocarpine, leading to its limited effect on the therapeutic effectiveness of APAC. (2) In terms of drug effect, brinzolamide has been known to reduce IOP by less than $10 \mathrm{mmHg}$ in the treatment of open-angle glaucoma.[11] Corneal edema may affect brinzolamide absorption to some extent, and the frequent use of pilocarpine may elute brinzolamide and reduce its concentration.[12] However, in this study, the initial IOP of APAC was greater than $40 \mathrm{mmHg}$, which made the IOP-lowering effect of brinzolamide seem smaller. (3) In terms of sequential treatment, the time to achieve successful treatment is an important index for evaluating the efficiency of emergency rescue. Both ACP and ALPI significantly reduced the IOP more rapidly than brinzolamide did. Six hours after beginning treatment, brinzolamide had just reached the peak of drug action, and ACP stepped in. However, subsequent remission depended more on pupil constriction than reduction of IOP. In other words, the application of ACP and ALPI reduced the need for brinzolamide.

The target IOP varies during the different stages of different types of glaucoma.[13] In this study, the target IOP for successful treatment was set at $<30 \mathrm{mmHg}$ instead of $21 \mathrm{mmHg}$ because this criterion was used to determine whether the treatment plan needed to be adjusted. When the corneal edema subsided and the pupil diameter narrowed to less than $4 \mathrm{~mm}$, the IOP generally continued to decline. Even if the IOP 
had not dropped below $21 \mathrm{mmHg}$, there was no need for ACP or ALPI. In addition, we used pupil diameter and the degree of corneal edema as the criteria for successful treatment. The degree of corneal edema is often a reflection of the IOP levels, whereas a reduction in the pupil diameter represents the elimination of pupil block and atrial angle closure.

Emergency surgery is not the primary choice for APAC treatment because of its associated complications. The evidence is more abundant for antiglaucoma agents, ACP, and ALPl; the latter two are invasive treatments. Taking effectiveness and safety into consideration, the treatment scheme applied in this study was the sequential application of antiglaucoma agents, ACP, and ALPI. Pilocarpine eye drops were used throughout the entire treatment process. We demonstrated in this study that the vast majority of eyes emerged from the crisis within 12 hours.

The mechanisms of action of ACP and ALPI are also distinctly different: ACP alone reduces the IOP only temporarily, and APAC may recur if the pupil block is not resolved promptly.[14] Meanwhile, ALPI mechanically pulls open the closed drainage angle, thereby reestablishing aqueous outflow through the normal drainage channels. It may be associated with potential risks, such as corneal burn and corneal endothelial cell damage. Furthermore, laser access to the peripheral iris may be obscured in some patients by media opacities, such as corneal edema, arcus senilis, or pterygium. There is no report on APAC treated with ALPI alone or ACP alone. In our study, ACP was able to rapidly reduce corneal edema and provide transparent optical pathways for ALPI.

In this research, the patients generally, but not always, had improved VA after treatment. Some patients were in remission, but their VA did not improve because of unrecovered corneal opacity. However, while other patients had high IOP levels, their corneas were transparent, and their VA was good. More research is needed on the specific mechanisms that affect VA.[15]

The study still had some limitations. First, although brinzolamide is commonly used to treat PACG, openangle glaucoma is a main indication for it, which may have affected the results. Second, there is no accurate, accepted test for visual field, but a patient's visual field is a direct indicator of disease progression. Therefore, we evaluated the therapeutic effect of the drug by the change of IOP. Finally, even without considering the cost burden, the application of brinzolamide is also associated with the following problems: APAC mainly occurs in the middle/elderly population, for whom the adverse reactions of electrolyte disorder have always been a clinical risk that cannot be ignored.[16] During the follow-up observation after achieving control of APAC, the false appearance of low IOP caused by brinzolamide often affected the evaluation of the disease.

Based on this study's results, we believe that in hospitals where ACP and ALPI can be performed, adding brinzolamide may be appropriate for patients with a first episode of APAC. However, brinzolamide should not be used as a first-line drug.

\section{Declarations}




\section{Competing interests}

All of the authors had no any personal, financial, commercial, or academic conflicts of interest separately.

\section{Funding}

Sources of support that require acknowledgment: The project received the financial support from Foundation of Hebei Public Health Department.

\section{Authors' contributions}

Guang Chen and Xinming Peng conceived of the study, and Jun Li, Peng Chen participated in its design and coordination and Jing Wang helped to draft the manuscript. All authors read and approved the final manuscript.

\section{Availability of data and materials}

All data generated or analyzed during this study are included in this published article.

\section{Animal Research}

Not applicable

\section{Ethics approval and consent to participate}

This study was conducted in accordance with the Declaration of Helsinki and approved by the ethics committee of Affiliated Hospital of Hebei University. All patients consent to participate in this study.

\section{Consent for publication}

Not applicable

\section{References}

1. Quigley HA, Broman AT. The number of people with glaucoma worldwide in 2010 and 2020. Br J Ophthalmol, 2006. 90(3): p. 262-7.

2. Prum JrBE, Herndon JrLW, Moroi SE, Mansberger SL, Stein JD, Lim MC, Rosenberg LF, Gedde SJ, Williams RD. Primary Angle Closure Preferred Practice Pattern((R)) Guidelines. Ophthalmology, 2016. 123(1): p. P1-P40.

3. Nongpiur ME, Aung T. Mechanisms underlying acute angle closure. Clin Exp Ophthalmol, 2017. 45(4): p. 331-332.

4. Pu H, Wang Y, Wei Q, Ma HJ, Hu PP, Li SL, Pang HB, Tian YH, Wang K. Decision-making Impairments in Primary Angle-closure Glaucoma Patients. Chin Med J (Engl), 2017. 130(12) p 1424-1428 
5. Seah SK, Foster PJ, Chew PT, Jap A, Oen F, Fam HB, Lim AS. Incidence of acute primary angle-closure glaucoma in Singapore. An island-wide survey. Arch Ophthalmol, (1997) 115(11): p. 1436-40.

6. Zhao Y, Fu JL, Li YL, Li P, Lou FL. Epidemiology and clinical characteristics of patients with glaucoma: An analysis of hospital data between 2003 and 2012. Indian J Ophthalmol, 2015. 63(11): p. $825-31$.

7. Tham YC, Li X, Wong TY, Quigley HA, Aung T, Cheng CY. Global prevalence of glaucoma and projections of glaucoma burden through 2040: a systematic review and meta-analysis. Ophthalmology, 2014. 121(11): p. 2081-90.

8. Singh P, Rijal AP. Effectivity of Nd Yag PI in treatment of acute primary angle closure glaucoma. Nepal Med Coll J, 2014. 16(1): p. 45 - 9.

9. Lai J, Choy BN, Shum JW. Management of Primary Angle-Closure Glaucoma. Asia Pac J Ophthalmol (Phila), 2016. 5(1) p 59-62

10. Chan PP, Pang JC, Tham CC. Acute primary angle closure-treatment strategies, evidences and economical considerations. Eye (Lond), (2019) 33(1): p. 110-119.

11. Li TJ, Lindsley K, Rouse B, Hong H, Shi QY, Friedman DS, et al. Comparative Effectiveness of FirstLine Medications for Primary Open-Angle Glaucoma: A Systematic Review and Network Metaanalysis. Ophthalmology, 2016. 123(1): p. 129 - 40.

12. Pisella PJ, Pouliquen P, Baudouin C. Prevalence of ocular symptoms and signs with preserved and preservative free glaucoma medication. Br J Ophthalmol, (2002) 86(4): p. 418 - 23.

13. Sihota R, Angmo D, Ramaswamy D, Dada $T$. Simplifying "target" intraocular pressure for different stages of primary open-angle glaucoma and primary angle-closure glaucoma. Indian J Ophthalmol, 2018. 66(4) p 495-505

14. Lam DSC, Chua JKH, Tham CCY, Lai JSM. Efficacy and safety of immediate anterior chamber paracentesis in the treatment of acute primary angle-closure glaucoma: a pilot study. Ophthalmology, 2002. 109(1) p 64-70

15. Zhang XL, Liu YM, Wang W, Chen SD, Li F, Huang WB, Aung T, Wang NL.Why does acute primary angle closure happen? Potential risk factors for acute primary angle closure. Surv Ophthalmol, 2017. 62(5) p 635-647

16. Liu YZ, Zhao JY, Zhong XY, Wei QM, Huang YL. Efficacy and Safety of Brinzolamide as Add-On to Prostaglandin Analogues or beta-Blocker for Glaucoma and Ocular Hypertension: A Systematic Review and Meta-Analysis. Front Pharmacol, 2019. 10: $p 679$

\section{Tables}




\begin{tabular}{|llll|}
\hline \multicolumn{4}{|l|}{ Table 1.Demographics of the patients } \\
Items & brinzolamide group & placebo group & Overall \\
\hline Number of eyes & 63 & 68 & 131 \\
\hline Number of patients & 61 & 64 & 125 \\
\hline Male/female & $11 / 50$ & $15 / 49$ & $26 / 99$ \\
\hline Mean age \pm SD \years $\Downarrow$ & $65.5 \pm 9.3$ & $63.9 \pm 8.7$ & $64.7 \pm 8.9$ \\
\hline Right/Left eye & $36 / 27$ & $35 / 33$ & $71 / 60$ \\
\hline
\end{tabular}

SD: standard deviation

\begin{tabular}{|c|c|c|c|c|}
\hline \multirow[t]{2}{*}{ Times } & \multicolumn{2}{|l|}{ Brinzolamide group } & \multicolumn{2}{|l|}{ Placebo group } \\
\hline & mean IOP $\pm S D(m m H g)$ & $P$ value & mean IOP $\pm S D(m m H g)$ & $P$ value \\
\hline Baseline & $55.14 \pm 6.31$ & - & $53.57 \pm 5.92$ & - \\
\hline 6 hours & $39.62 \pm 16.97$ & $\varangle 0.001$ & $36.49 \pm 16.43$ & $\bowtie 0.001$ \\
\hline 12 hours & $18.69 \pm 8.80$ & $\otimes 0.001$ & $19.03 \pm 9.98$ & $\nabla 0.001$ \\
\hline 24 hours & $17.83 \pm 7.97$ & $\otimes 0.001$ & $18.15 \pm 8.92$ & $\nabla 0.001$ \\
\hline
\end{tabular}

SD: standard deviation

\begin{tabular}{|llll|}
\hline \multicolumn{3}{|c|}{ Table 3.The mean reduction of the IOP at each time interval } \\
\hline Times & Brinzolamide group $(\mathrm{mmHg})$ & Placebo group $(\mathrm{mmHg})$ & P value \\
\hline 6 hours & $15.52 \pm 14.62$ & $17.09 \pm 15.10$ & 0.526 \\
\hline 12 hours & $36.45 \pm 9.12$ & $34.54 \pm 10.66$ & 0.206 \\
\hline 24 hours & $37.32 \pm 8.95$ & $35.43 \pm 9.70$ & 0.130 \\
\hline
\end{tabular}




\begin{tabular}{|llll|}
\hline \multicolumn{4}{|l|}{ Table 4. The success rates at different follow up times } \\
\hline Times & Brinzolamide group & Placebo group & P value \\
\hline 6 hours & $38.1 \%(24 / 63)$ & $44.12 \%(30 / 68)$ & 0.651 \\
\hline 12 hours & $93.65 \%(59 / 63)$ & $89.71 \%(61 / 68)$ & 0.865 \\
\hline 24 hours & $95.24 \%(60 / 63)$ & $92.65 \%(63 / 68)$ & 0.913 \\
\hline
\end{tabular}

Table 5. The amount of mannitol, patients taken ACP or ALPI in the two groups

\begin{tabular}{|llll|}
\hline Items & Brinzolamide group & Placebo group & P value \\
\hline amount of mannitol & $380.95 \pm 133.63$ & $367.65 \pm 125.71$ & 0.611 \\
\hline patients taken ACP & $26 / 60$ & $22 / 63$ & 0.527 \\
\hline patients taken ALPI & $8 / 60 \quad 10 / 63$ & & 0.731 \\
\hline
\end{tabular}

\section{Figures}

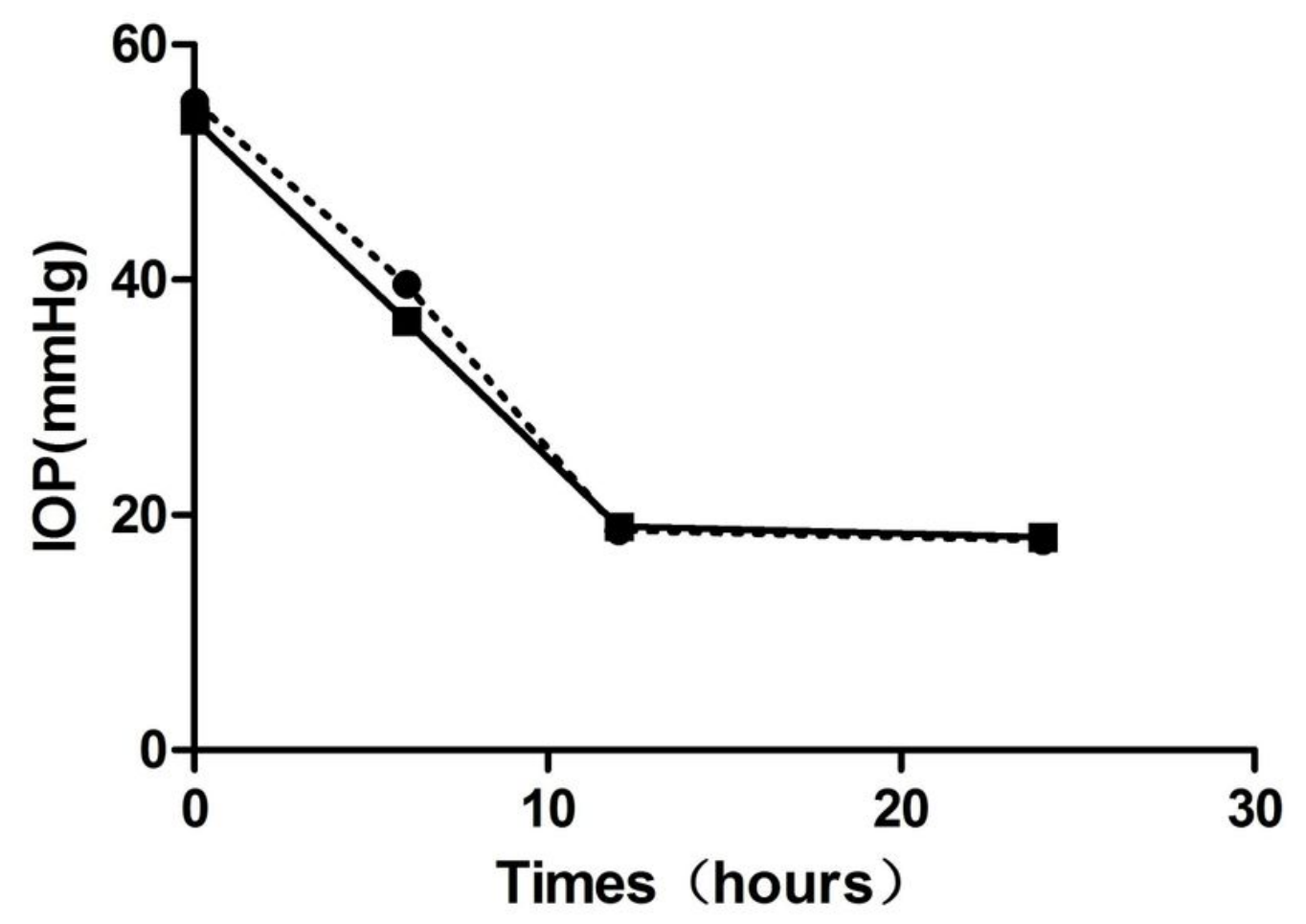

Figure 1

Mean IOP at different follow up times $(\mathrm{mmHg})$

- - Brinzolamide

$\rightarrow$ Placebo 


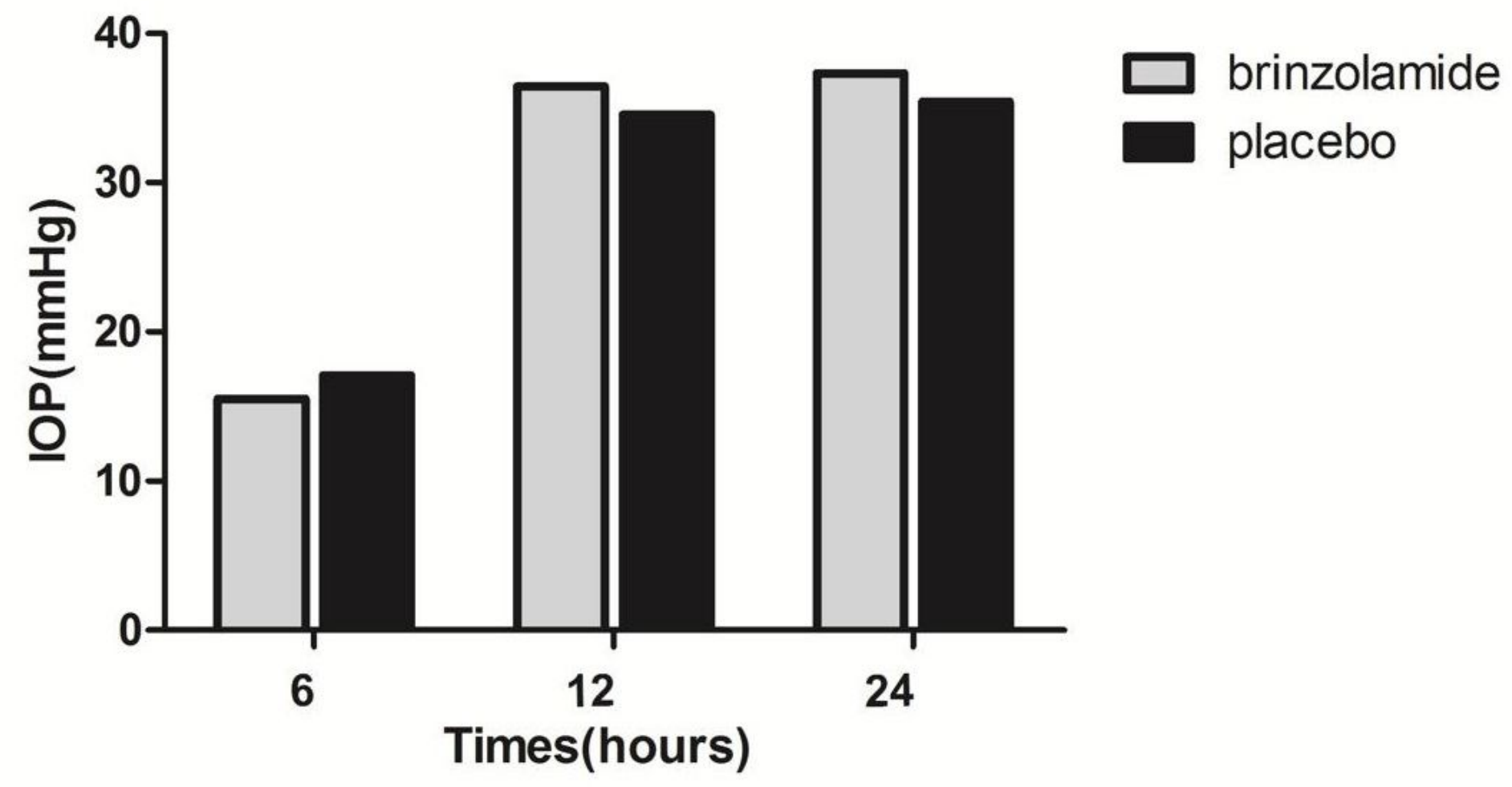

Figure 2

The mean reduction of the IOP at each time interval $(\mathrm{mmHg})$

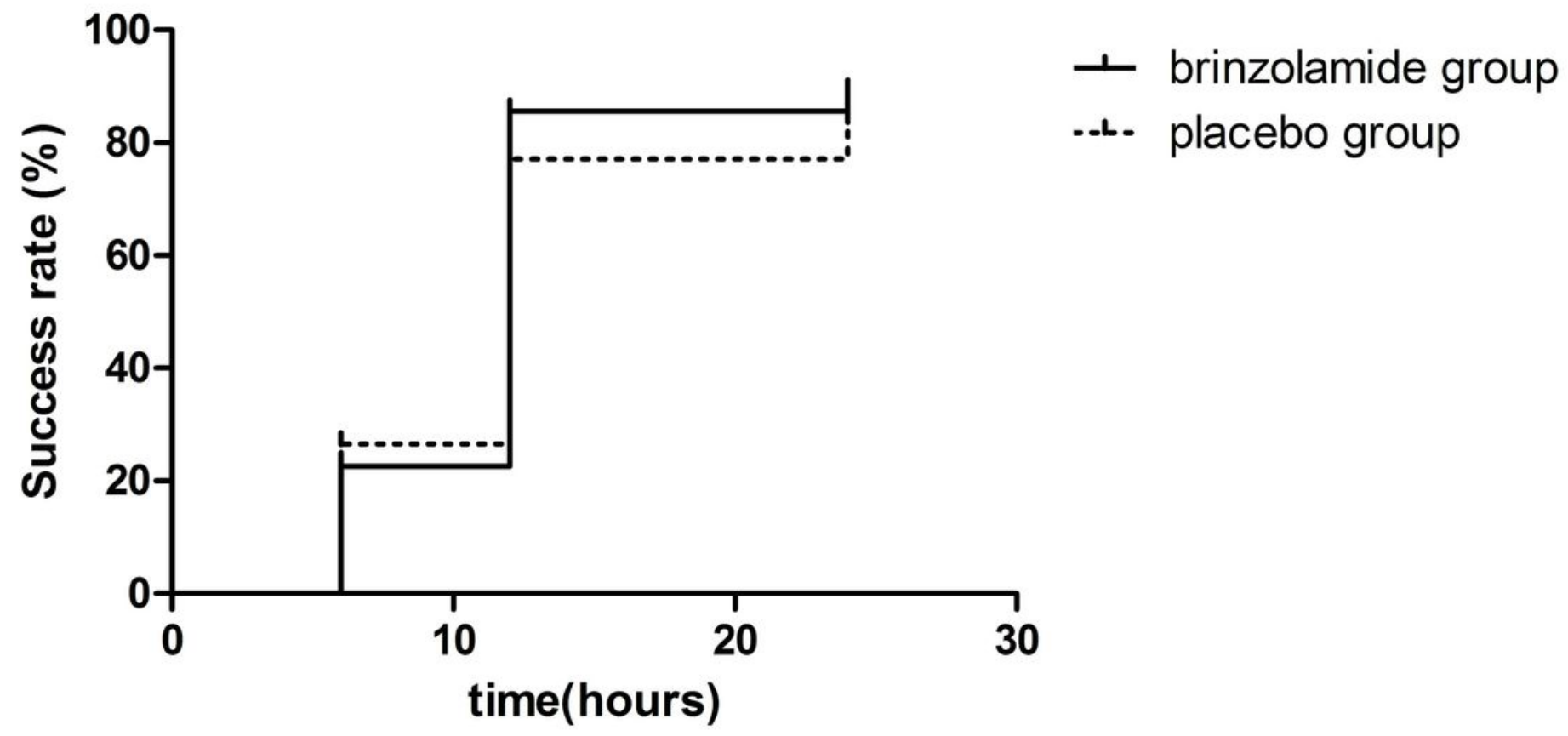

Figure 3

The success rates at different follow up times 